\title{
Radial pulse transit time is an index of arterial stiffness
}

\author{
Yong-Liang Zhang ${ }^{1,2}$, Ying-Ying Zheng ${ }^{1,2}$, Zu-Chang $\mathrm{Ma}^{1}$ and Yi-Ning Sun ${ }^{1}$
}

Aortic pulse wave velocity, calculated from pulse transit time (PTT), is often used as an indicator of arterial stiffness and suggested to be standardized for heart rate (HR). This study aimed to determine whether PTT obtained directly from radial arterial waveforms could be used to assess arterial stiffness and the effect of HR on it. Measurements of anthropometric parameters, blood pressure (BP) and radial PTT were taken in 266 apparently healthy adults (113 men and 153 women; age 18-78 years). BP and radial PTT were measured in a subgroup of 11 young subjects (seven men and four women, age 24-35 years) in a 3-month follow-up study, which aimed to investigate the effect of HR changes. Radial PTT was significantly higher in men compared with women $(0.116 \pm 0.022 \mathrm{~s}$ compared with $0.103 \pm 0.031 \mathrm{~s}, P<0.001)$. It was inversely related to age in men and women ( $r=-0.838$ and $r=-0.804$, respectively, $P<0.01$ for both). Multiple regression analysis showed that HR was a potent predictor of radial PTT in addition to age, sex and systolic BP. There was no significant change in radial PTT when HR ranged from 60 to 75 b.p.m. A significant decrease was found in radial PTT when HR was up to 80 b.p.m. $(P<0.01$ ). These findings indicate that the simple and easily obtainable radial PTT could be a useful index of arterial stiffness, and HR changes should be considered when it is up to 80 b.p.m.

Hypertension Research (2011) 34, 884-887; doi:10.1038/hr.2011.41; published online 19 May 2011

Keywords: arterial stiffness; blood pressure; heart rate; pulse transit time

\section{INTRODUCTION}

Aortic pulse wave velocity (PWV), calculated from pulse transit time (PTT), has become an indicator of arterial stiffness. ${ }^{1-3}$ Aging is accompanied by increased arterial stiffness and aortic PWV, ${ }^{4,5}$ leading to a decrease in PTT. This suggests that arterial stiffness might be assessed by PTT. The general method for obtaining PTT is to calculate the time delay between the foot of pressure upstroke at carotid and femoral arteries. ${ }^{6-8}$ Although the method is useful in research and clinical laboratories, attempts have been made to simplify the measurement procedure, which result in determination of PTT from a single pulse measurement. In the study of Qasem et al., ${ }^{9}$ waveform decomposition of the aortic pressure wave into forward and reflected waves and comparison between them have been used to estimate PTT in the aorta. PTT has also been estimated from the time of the inflection point in the aorta. ${ }^{10}$ For aortic pressure pulses are estimated from radial tonometry pulses with the use of a generalized transfer function, ${ }^{7,11,12}$ it raises the possibility that PTT could be derived directly from radial arterial pulses and provided equivalent information to aortic PTT. The study of Millasseau et al. ${ }^{13}$ suggested that similar information on the aortic pressure wave could be obtained directly from the radial pulse.

In the present study, we proposed a novel noninvasive method to obtain PTT from a single radial pressure waveform. This method estimated radial PTT by calculating the time interval between the first and second systolic peaks of the radial pulse wave (Figure 1), which should not be transformed to the aortic pressure wave first. In previous studies, PTT was influenced by heart rate $(\mathrm{HR})^{14-16}$ and blood pressure (BP). ${ }^{17}$ The effect of HR on the propagation of pulse waves has been found not only in central arteries, ${ }^{14}$ but also in small peripheral arteries ${ }^{16}$ and the microcirculation. ${ }^{15}$ Bernjak et al. ${ }^{15}$ showed that PTT through the microcirculation was negatively correlated with HR. Our hypothesis was that values of radial PTT might also be influenced by HR. Therefore, the aim of the study was to investigate the relationship between radial PTT and age in healthy subjects and whether or not HR changes influenced radial PTT.

\section{METHODS}

\section{Subjects}

A total of 266 healthy adults (113 men and 153 women) aged $18-78$ years ( $37.9 \pm 18.9$ years) underwent the measurements of anthropometric parameters (height, body mass), BP and radial PTT. A subgroup of 11 young subjects (seven men and four women) aged 24-35 years (27.3 \pm 3.2 years) had a 3-month follow-up study, in which each subject took the measurements of $\mathrm{BP}$ and radial PTT twice every day (at 0800 and $1700 \mathrm{~h}$, separately). All subjects were free of cardiovascular disease and vasoactive medications, as assessed by a medical history questionnaire. Written informed consent for the procedure was obtained from each subject, and the study was approved by the local Institutional Review Board.

\section{Measurements}

Before testing, subjects were asked to fast and abstain from cigarettes and alcohol for $3 \mathrm{~h}$ or more. After taking the measurements of height and body mass and resting in the sitting position for $5 \mathrm{~min}$ in a quiet, temperature-

${ }^{1}$ Institute and Intelligent of Machines, Chinese Academy of Sciences, Hefei, Anhui, PR China and ${ }^{2}$ Department of Automation, University of Science and Technology of China, Hefei, Anhui, PR China

Correspondence: Professor Y-N Sun, Institute and Intelligent of Machines, Chinese Academy of Sciences, 350 Shushanghu Road, Hefei, Anhui 230031, PR China.

E-mail: lake.ronin@gmail.com

Received 13 July 2010; revised 21 January 2011; accepted 20 February 2011; published online 19 May 2011 


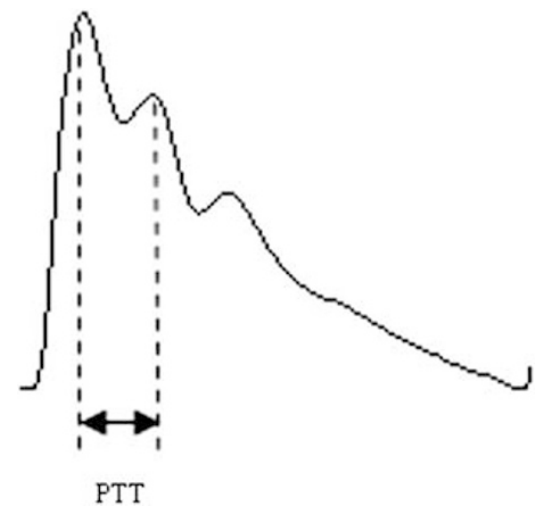

Figure 1 Calculated pulse transit time (PTT) by the time interval between the first and second peaks of the radial pulse wave.

controlled room, BP was measured on the right wrist using the oscillometric method (HEM-6000; Omron Healthcare, Kyoto, Japan).

Radial PTT was derived using an applanation tonometry-based automated vascular testing device (IIM-2010A; Institute of Intelligent of Machines, Hefei, China). The size of the device is $26 \times 20 \times 8 \mathrm{~cm}$ and its weight is $1.2 \mathrm{~kg}$. The arterial applanation tonometer was held on the left radial artery while pressure against the radial artery was obtained and digitized into digital volume pulse using a 12-bit analogue-to-digital converter with a sampling frequency of $200 \mathrm{~Hz}$. The magnification of digital volume pulse was modulated automatically to obtain an optimal radial arterial waveform. Consistent radial arterial waveforms were recorded for $12 \mathrm{~s}$, the first and second systolic peaks of which were automatically detected by using algorithms of the device based on a bandpass filtering $(0.02-80 \mathrm{~Hz})$ and fourth derivatives. The time intervals between the first and second systolic peaks of radial pulse waves were calculated and averaged value was reported as radial PTT.

\section{Statistical analysis}

All values were expressed as means \pm s.d., if not specified. The differences between men and women were evaluated by analysis of variance. Multiple regression analysis using enter method for radial PTT was performed with the following parameters: age, sex, systolic BP (SBP), diastolic BP (DBP), HR, height and body mass. An analysis of covariance was performed with radial PTT as the dependent variable and HR as covariate. The effect of HR on BP and radial PTT was tested by one-way, within-subjects analysis of variance with repeated measurements. A least significant difference post hoc analysis, with a least significant difference test was performed to determine which levels of HR led to significant changes in PTT. A probability value $P<0.05$ was considered to indicate statistical significance.

\section{RESULTS}

\section{Radial PTT and Age}

As shown in Table 1, height, body mass, PTT, SBP and DBP were lower in women than in men $(P<0.05)$, whereas $\mathrm{HR}$ was higher in women than in men $(P<0.05)$. Figure 2 illustrated the relationship between radial PTT and age. Radial PTT was inversely related to age in men and women $(r=-0.838$ and $r=-0.804$, respectively, $P<0.01$ for both). Age-related changes in radial PTT were significant $(P<0.001)$. The values of radial PTT were expressed as mean values in each decade of life and shown in (Figure 2b). There were significant age-dependent decreases in radial PTT from 20 years to 40 years in men and women $(P<0.01$ for both). Multiple regression analysis revealed that radial PTT was significantly associated with age in addition to sex, HR and SBP, whereas DBP, height and body mass were not associated with radial PTT (Table 2). The analysis of covariance analysis further showed there was a significant difference in radial PTT with a wide age range $(P<0.001$, Table 3$)$.
Table 1 Selected characteristics of the study participants $(n=266)$

\begin{tabular}{lcc}
\hline Variables & Male & Female \\
\hline Age (years) & $38.2 \pm 18.4$ & $38.3 \pm 19.4$ \\
Height (cm) & $170.7 \pm 4.7$ & $160.8 \pm 4.5^{* *}$ \\
Body mass (kg) & $65.6 \pm 9.2$ & $54.3 \pm 8.2^{* *}$ \\
HR (beats min $\left.{ }^{-1}\right)$ & $70.9 \pm 8.0$ & $73.4 \pm 8.4^{*}$ \\
PTT (s) & $0.117 \pm 0.024$ & $0.103 \pm 0.031^{* *}$ \\
SBP (mmHg) & $117.8 \pm 9.2$ & $110.8 \pm 8.7^{* *}$ \\
DBP (mmHg) & $79.1 \pm 7.6$ & $74.3 \pm 7.7^{* *}$ \\
\hline
\end{tabular}

Abbreviations: $\mathrm{DBP}=$ diastolic blood pressure; $\mathrm{PTT}=$ pulse transit time; $\mathrm{HR}=$ heart rate; $\mathrm{SBP}=$ systolic blood pressure.

Values are means \pm s.d.

${ }^{*} P<0.05 ;{ }^{* *} P<0.001$, vs. male.

\section{Effect of HR changes on BP and radial PTT}

In the second experiment, the changes of HR contained the range from 59-82 b.p.m. for all subjects. The following five different levels $(60,65,70,75$ and 80 b.p.m.) were selected and the values of radial PTT at the HR most close to the selected level were entered the study.

The effect of HR on BP was illustrated in (Figure 3a). SBP and DBP remained stable on average (not significant for HR changes). The values of radial PTT at each HR level (60, 65, 70, 75 and 80 b.p.m.) were $0.144 \pm 0.008,0.143 \pm 0.006,0.143 \pm 0.009,0.138 \pm 0.008$ and $0.134 \pm 0.007 \mathrm{~s}$, respectively. A post hoc test showed that significant differences in radial PTT were just observed between 80 b.p.m. and each other HR levels, except 75b.p.m. (60: $P=0.003,65$ : $P=0.005$ and 70: $P=0.007$, respectively). Radial PTT obtained for consecutive HR values were not statistically different.

\section{DISCUSSION}

The studies described by Lydakis et al. ${ }^{10}$ and McEniery et al. ${ }^{18}$ have demonstrated the proof of the concept that PTT could be estimated from the measurement of a single aortic pulse wave. They showed that the inflection point could be identified by waveform analysis of the aortic pressure pulse. The present study extended this concept by estimating radial PTT as the time interval between the first and second systolic peaks of the radial pulse wave (Figure 1), which meant PTT was determined directly and easily from the radial pulse wave rather than a transformed aortic pulse. The result of the study has showed a significant association between radial PTT and age, both in men $(r=-0.838, P<0.01)$ and women $(r=-0.804, P<0.01)$. Furthermore, there was an age-related decrease in radial PTT in healthy men and women aged 18-78 years. In previous studies, arterial stiffness was increased with aging. ${ }^{4,5}$ Therefore, these findings indicates that the simple and easily obtainable radial PTT is age-dependent and could be a useful index of arterial stiffness.

As shown in Figure 2b, radial PTT decreased significantly with age from 20 years to 40 years, whereas insignificantly in older subjects. A possible reason for the phenomenon might be the movement towards the periphery in reflection site, which led to a longer traveling distance of the reflected wave. This is in accordance with the result of several previous studies. ${ }^{19-21}$ Mitchell et al. ${ }^{19}$ showed that, with aging, the aortic PWV increased, whereas the time of return of the reflected wave decreased only little. These authors, therefore, speculated that the reflection site was moving toward the periphery.

Multiple regression analysis revealed that age, HR, sex and SBP were entered into the model as independent predictors of radial PTT (Table 2), which were also contributors to $\mathrm{PWV}^{22}$ and $\mathrm{AI}^{23,24}$ Furthermore, the result of the analysis of covariance with dependent variable of radial PTT and covariate of HR showed a significant 

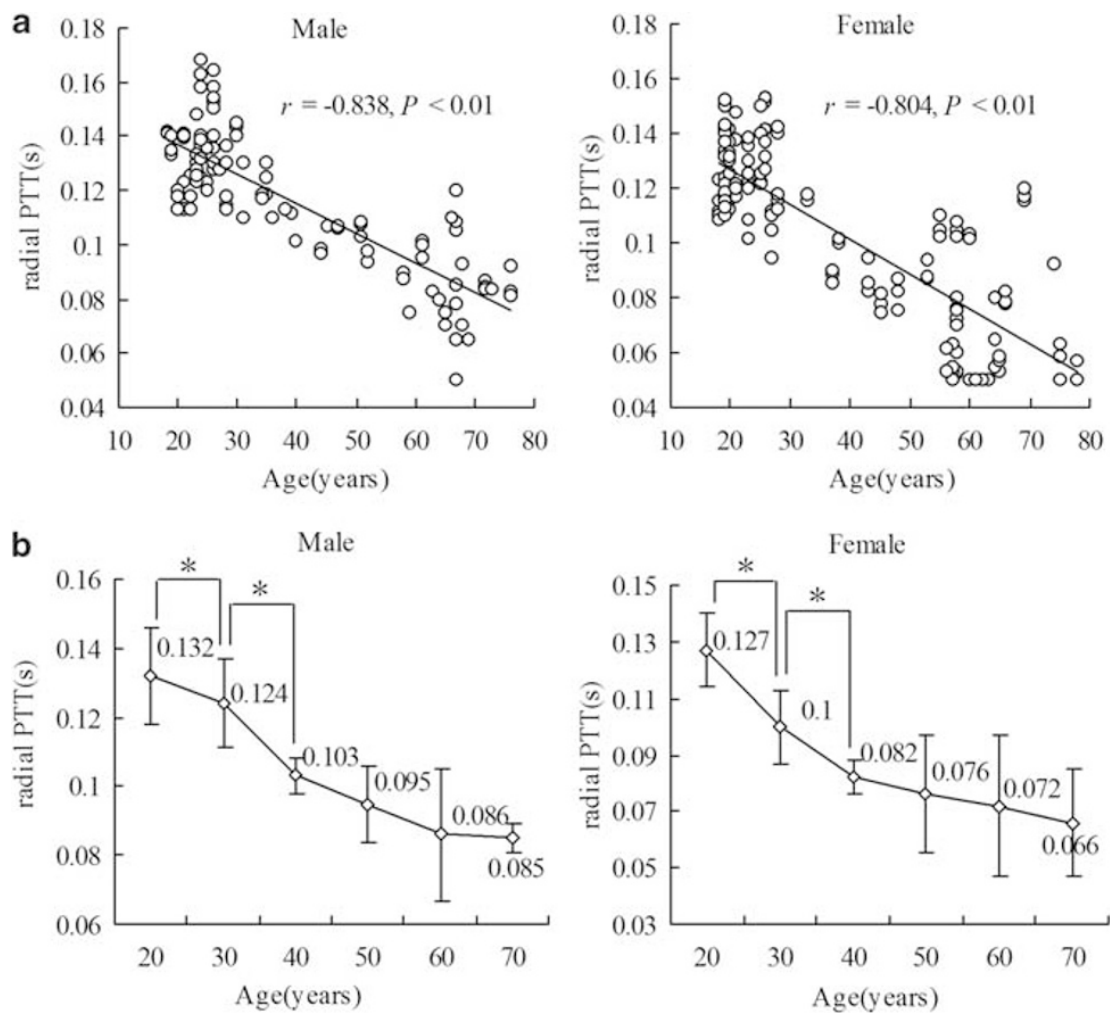

Figure 2 Correlation between radial pulse transit time (PTT) and age in men and women (a) and changes in radial PTT in men and women for each decade (b). Values are means \pm s.d. ${ }^{*} P<0.01$ between age groups.

Table 2 Multiple regression analyses for radial pulse transit time $(n=266)$

\begin{tabular}{lrrr}
\hline Variables & \multicolumn{1}{c}{$\boldsymbol{t}$} & \multicolumn{1}{c}{ P-value } \\
\hline Age & -0.754 & -20.460 & $<0.001$ \\
Sex & -0.154 & -3.018 & 0.003 \\
SBP & 0.124 & 2.273 & 0.024 \\
DBP & -0.093 & -1.694 & 0.091 \\
HR & -0.109 & -3.024 & 0.003 \\
Body mass & -0.061 & -1.210 & 0.227 \\
Height & 0.110 & 1.880 & 0.061 \\
\hline
\end{tabular}

Abbreviations: $\mathrm{DBP}=$ diastolic blood pressure; $\mathrm{HR}=$ heart rate; $\mathrm{SBP}=$ systolic blood pressure.

difference in radial PTT with a wide age range, even if the effect of HR was considered.

Among these factors, HR is a complicated one, because it influences not only timing and strength of the reflected wave, ${ }^{14,25}$ but also other contributors of radial PTT such as $\mathrm{BP}^{26}$ In our second experiment, radial PTT changed only little when HR ranged from 60 to 75 b.p.m., whereas it decreased significantly when HR was up to 80 b.p.m. (Figure 3). PTT is calculated as traveling distance divided by PWV. In the study by Lantelme et al., 22 PWV increased significantly with HR, when HR was more than 80 b.p.m. This may be the reason why radial PTT had no significant changes until HR was up to 80 b.p.m.

There have been other attempts at obtaining stiffness index from waveform analysis of a single peripheral pulse. Millasseau et al. ${ }^{27}$ reported that a time interval, assumed to be related to aortic wave transmission, was obtained by feature extraction of the digital volume pulse, and then a stiffness index (equivalent to a PWV parameter) was
Table 3 Analysis of covariance for radial PTT with HR as covariate $(n=266)$

\begin{tabular}{lcr}
\hline Variables & $F$ & P-value \\
\hline Age & 20.344 & $<0.001$ \\
HR & 13.141 & $<0.001$ \\
\hline
\end{tabular}

Abbreviations: $\mathrm{HR}=$ heart rate; $\mathrm{PTT}=$ pulse transit time.

obtained by the ratio of height and this time interval. Although a significant correlation was found between the calculated stiffness index and measured aortic PWV, a possible source of error could arise from the estimation of traveling distance of the reflected wave from height. In the present study, therefore, we applied a similar analysis to the radial pulse just to ascertain whether any association existed between radial PTT and age. However, though a significant association was found between radial PTT and age, the information from radial PTT could not be expected to be identical to PWV, for the shape of the radial pulse was influenced by various factors. ${ }^{28-30}$

In summary, PTT obtained directly from radial arterial waveforms was significantly associated with age in healthy adult with a wide age range. The effect of HR changes on radial PTT was insignificant until it was up to 80 b.p.m. These findings support that the simple and easily obtainable radial PTT could be a useful parameter of arterial stiffness, and HR changes should be taken into consideration when it is up to 80 b.p.m.

A limitation of the study is the lack of the objective measurement of PTT. Radial PTT obtained using the method proposed in the study was not evaluated against PTT determined from two independent recordings. However, radial PTT was inversely related to age, both in 

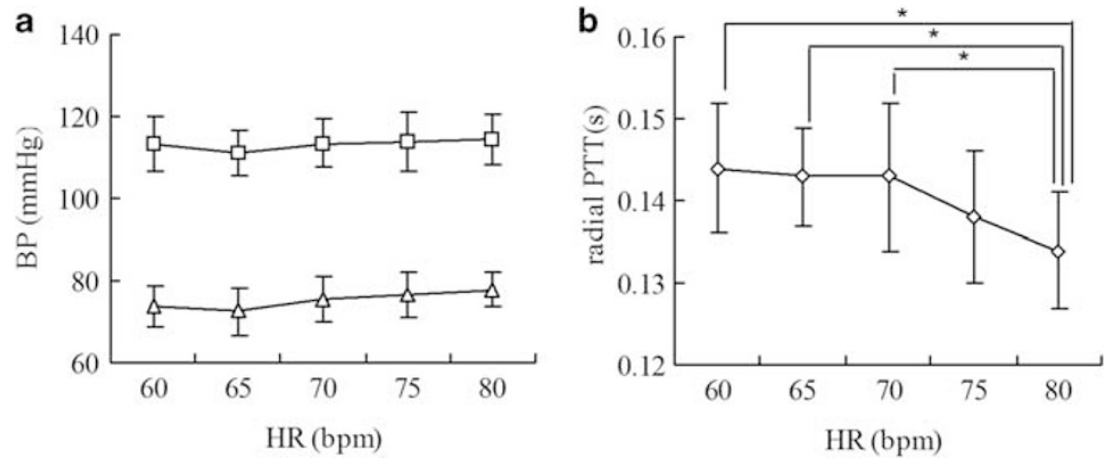

Figure 3 Changes in systolic BP (SBP, squares), diastolic BP (DBP, triangles) (a) and radial pulse transit time (PTT) for each heart rate (HR) level (b). $\mathrm{BP}$, blood pressure. Values are means \pm s.d. ${ }^{*} P<0.01$ between HR levels.

men and women, indicating that it could be used as an index of arterial stiffness. In addition, radial PTT is very useful in research, because it is simple and easily obtainable.

\section{CONFLICT OF INTEREST}

The authors declare no conflict of interest.

\section{ACKNOWLEDGEMENTS}

We are grateful to Chi-Wen Lung for article review.

1 Nichols WW. Clinical measurement of arterial stiffness obtained from noninvasive pressure waveforms. Am J Hypertens 2005; 18: 3S-10S.

2 Oliver JJ, Webb DJ. Noninvasive assessment of arterial stiffness and risk of atherosclerotic events. Arterioscler Thromb Vasc Biol 2003; 23: 554-566.

3 Meaume S, Benetos A, Henry OF, Rudnichi A, Safar ME. Aortic pulse wave velocity predicts cardiovascular mortality in subjects $>70$ years of age. Arterioscler Thromb Vasc Biol 2001; 21: 2046-2050.

4 Koivistoinen T, Koobi T, Jula A, Hutri-Kahonen N, Raitakari OT, Majahalme S, Kukkonen-Harjula K, Lehtimaki T, Reunanen A, Viikari J, Turjanmaa V, Nieminen T, Kahonen M. Pulse wave velocity reference values in healthy adults aged $26-75$ years. Clin Physiol Funct Imaging 2007; 27: 191-196.

5 Reusz GS, Cseprekal O, Temmar M, Kis E, Cherif AB, Thaleb A, Fekete A, Szabo AJ, Benetos A, Salvi $P$. Reference values of pulse wave velocity in healthy children and teenagers. Hypertension 2010; 56: 217-224.

6 DeLoach SS, Townsend RR. Vascular stiffness: its measurement and significance for epidemiologic and outcome studies. Clin J Am Soc Nephrol 2008; 3: 184-192.

7 Pauca AL, O'Rourke MF, Kon ND. Prospective evaluation of a method for estimating ascending aortic pressure from the radial artery pressure waveform. Hypertension 2001; 38: 932-937.

8 Asmar RG, Topouchian JA, Benetos A, Sayegh FA, Mourad JJ, Safar ME. Non-invasive evaluation of arterial abnormalities in hypertensive patients. J Hypertens 1997; 15: S99-S107.

9 Qasem A, Avolio A. Determination of aortic pulse wave velocity from waveform decomposition of the central aortic pressure pulse. Hypertension 2008; 51: 188-195.

10 Lydakis C, Momen A, Blaha C, Gugoff S, Gray K, Herr M, Leuenberger UA, Sinoway LI. Changes of central haemodynamic parameters during mental stress and acute bouts of static and dynamic exercise. J Hum Hypertens 2008; 22: 320-328.

11 Chen CH, Nevo E, Fetics B, Pak PH, Yin FCP, Maughan WL, Kass DA. Estimation of central aortic pressure waveform by mathematical transformation of radial tonometry pressure-validation of generalized transfer function. Circulation 1997; 95: 1827-1836.

12 Sharman JE, Lim R, Qasem AM, Coombes JS, Burgess MI, Franco J, Garrahy P, Wilkinson IB, Marwick TH. Validation of a general ized transfer function to noninvasively derive central blood pressure during exercise. Hypertension 2006; 47: 1203-1208.
13 Millasseau SC, Patel SJ, Redwood SR, Ritter JM, Chowienczyk PJ. Pressure wave reflection assessed from the peripheral pulse: is a transfer function necessary? Hypertension 2003; 41: 1016-1020.

14 Papaioannou TG, Vlachopoulos CV, Alexopoulos NA, Dima I, Pietri PG, Protogerou AD, Vyssoulis GG, Stefanadis $\mathrm{Cl}$. The effect of heart rate on wave reflections may be determined by the level of aortic stiffness: clinical and technical implications. Am J Hypertens 2008; 21: 334-340.

15 Bernjak A, Stefanovska A. Pulse transit times to the capillary bed evaluated by laser Doppler flowmetry. Physiol Meas 2009; 30: 245-260.

16 Drinnan MJ, Allen J, Murray A. Relation between heart rate and pulse transit time during paced respiration. Physiol Meas 2001; 22: 425-432.

17 Wong YM, Zhang YT. The effects of exercises on the relationship between pulse transit time and arterial blood pressure. Conf Proc IEEE Eng Med Biol Soc 2005; 5: 5576-5578.

18 McEniery CM, Yasmin , Hall IR, Qasem A, Wilkinson IB, Cockcroft JR. Normal vascular aging: differential effects on wave reflection and aortic pulse wave velocity: the AngloCardiff Collaborative Trial (ACCT). J Am Coll Cardiol 2005; 46: 1753-1760.

19 Mitchell GF, Parise H, Benjamin EJ, Larson MG, Keyes MJ, Vita JA, Vasan RS, Levy D. Changes in arterial stiffness and wave reflection with advancing age in healthy men and women: the Framingham Heart Study. Hypertension 2004; 43: 1239-1245.

20 Westerhof BE, van den Wijngaard JP, Murgo JP, Westerhof N. Location of a reflection site is elusive: consequences for the calculation of aortic pulse wave velocity. Hypertension 2008; 52: 478-483.

21 Sugawara J, Hayashi K, Tanaka H. Distal shift of arterial pressure wave reflection sites with aging. Hypertension 2010; 56: 920-925.

22 Lantelme $\mathrm{P}$, Mestre C, Lievre M, Gressard A, Milon $H$. Heart rate: an important confounder of pulse wave velocity assessment. Hypertension 2002; 39: 1083-1087.

23 Kohara K, Tabara Y, Oshiumi A, Miyawaki Y, Kobayashi T, Miki T. Radial augmentation index: a useful and easily obtainable parameter for vascular aging. Am J Hypertens 2005; 18: 11S-14S

24 Sugawara J, Komine H, Hayashi K, Maeda S, Matsuda M. Relationship between augmentation index obtained from carotid and radial artery pressure waveforms. J Hypertens 2007; 25: 375-381.

25 Williams B, Lacy PS. Impact of heart rate on central aortic pressures and hemodynamics: analysis from the CAFE (Conduit Artery Function Evaluation) study: CAFEHeart Rate. J Am Coll Cardiol 2009; 54: 705-713.

26 Laurent P, Albaladejo P, Blacher J, Rudnichi A, Smulyan H, Safar ME. Heart rate and pulse pressure amplification in hypertensive subjects. Am J Hypertens 2003; 16: 363-370.

27 Millasseau SC, Kelly RP, Ritter JM, Chowienczyk PJ. Determination of age-related increases in large artery stiffness by digital pulse contour analysis. Clin Sci 2002; 103: 371-377.

28 Benthin M, Dahl P, Ruzicka R, Lindström K. Calculation of pulse-wave velocity using cross correlation-effects of reflexes in the arterial tree. Ultrasound Med Biol 1991; 17: 461-469.

29 Teng XF, Zhang YT. The effect of applied sensor contact force on pulse transit time. Physiol Meas 2006; 27: 675-684.

30 Liu JM, Wang JZ, Jin Y, Roethig HJ, Unverdorben M. Variability of peripheral arterial tonometry in the measurement of endothelial function in healthy men. Clin Cardiol 2009; 32: 700-704. 